\title{
BELLMAN EQUATIONS FOR TERMINAL UTILITY MAXIMIZATION WITH GENERAL BID AND ASK PRICES*
}

\author{
BY
}

TOMASZ ROGALA (WARSZAWA) AND LUKASZ S TE T T NER (WARSZAWA)

Abstract. In the paper we solve a system of Bellman equations for finite horizon continuous time terminal utility maximization problem with general càdlàg bid and ask prices. We assume that we have a restricted number of transactions at time moments we choose. The main result of the paper says that we can find a regular version of solutions to the system of Bellman equations, which enables us to find the form of nearly optimal strategies.

2010 AMS Mathematics Subject Classification: Primary: 93E20; Secondary: 91G10, 60G07.

Key words and phrases: Bellman equation, bid and ask prices, optimal stopping, terminal utility maximization.

\section{INTRODUCTION}

Assume we are given probability space $\left(\Omega, F,\left(F_{t}\right), P\right)$ satisfying usual conditions. Assume furthermore that $U$ is a continuous nondecreasing function defined on $R_{+}:=[0, \infty)$, and $\left(\underline{s}_{t}\right),\left(\bar{s}_{t}\right)$ are $\left(F_{t}\right)$-adapted càdlàg positive processes such that $\underline{s}_{t}<\bar{s}_{t}$ for $t \in[0, T]$. Let for $(x, y) \in R_{+}^{2}$

$$
A(x, y, \underline{s}, \bar{s})=\left\{(l, m) \in R_{+}^{2}: x+m \underline{s}-l \bar{s} \geqslant 0, y-m+l \geqslant 0\right\},
$$

and by $\mathcal{A}_{t}\left(x, y, \underline{s}_{t}, \bar{s}_{t}\right)$ we denote $F_{t}$-adapted random variables $(l, m)$ taking values in $A\left(x, y, \underline{s}_{t}, \bar{s}_{t}\right)$. Note that for $0<\underline{s}<\bar{s}$ and $(x, y) \in R_{+}^{2}$ the set $A(x, y, \underline{s}, \bar{s})$ is compact. Define the following sequence of parametrized processes for $(x, y) \in R_{+}^{2}$ and $t \in[0, T]$ :

* Research supported by NCN grant DEC-2012/07/B/ST1/03298. 


$$
\begin{aligned}
& V_{0}(x, y, \underline{s}, \bar{s}):=U(x+y \underline{s}), \\
& \bar{V}_{1}\left(x, y, \underline{s}_{t}, \bar{s}_{t}, t\right):=\operatorname{esssup}_{(l, m) \in \mathcal{A}_{t}\left(x, y, \underline{s}_{t}, \bar{s}_{t}\right)} E\left[V_{0}\left(x+m \underline{s}_{t}-l \bar{s}_{t}, y-m+l, \underline{s}_{T}, \bar{s}_{T}\right) \mid F_{t}\right], \\
& V_{1}(x, y, t):=\operatorname{esssup}_{t \leqslant \tau \leqslant T} E\left[\bar{V}_{1}\left(x, y, \underline{s}_{\tau}, \bar{s}_{\tau}, \tau\right) \mid F_{t}\right], \\
& \bar{V}_{2}\left(x, y, \underline{s}_{t}, \bar{s}_{t}, t\right):=\operatorname{ess} \sup _{(l, m) \in \mathcal{A}_{t}\left(x, y, \underline{s}_{t}, \bar{s}_{t}\right)} V_{1}\left(x+m \underline{s}_{t}-l \bar{s}_{t}, y-m+l, t\right), \\
& V_{2}(x, y, t):=\operatorname{ess}_{t \leqslant \tau \leqslant T} E\left[\bar{V}_{2}\left(x, y, \underline{s}_{\tau}, \bar{s}_{\tau}, \tau\right) \mid F_{t}\right],
\end{aligned}
$$

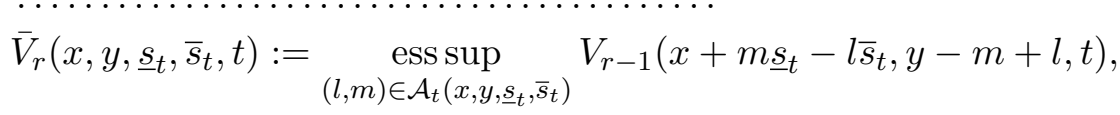

$$
\begin{aligned}
& V_{r}(x, y, t):=\operatorname{ess}_{t \leqslant \tau \leqslant T} E\left[\bar{V}_{r}\left(x, y, \underline{s}_{\tau}, \bar{s}_{\tau}, \tau\right) \mid F_{t}\right] .
\end{aligned}
$$

This system (L.2) of Bellman equations corresponds to the following portfolio optimization. We want to maximize terminal utility $U$ of the wealth process at time $T$, given adapted to $\left(F_{t}\right)$ bid and ask prices $\left(\underline{s}_{t}\right),\left(\bar{s}_{t}\right), \underline{s}_{t}<\bar{s}_{t}$, for $t \in[0, T]$. We assume that our portfolio position at time $t$ is characterized by the pair $\left(x_{t}, y_{t}\right)$, where $x_{t}$ is the amount located on the safe (bank) account while $y_{t}$ denotes a number of assets in our portfolio. We consider the case when we are not allowed to have short selling (both $x_{t}$ and $y_{t}$ variables should be nonnegative). Furthermore, we are allowed to have at most $r$ transactions over the time interval $[0, T]$. Consequently, our trading strategy will be in the form of sequence $\left(\tau_{i},\left(l^{i}, m^{i}\right)\right)$, where $0 \leqslant \tau_{1} \leqslant \tau_{2} \leqslant \ldots \leqslant \tau_{r} \leqslant T$, and $\left(l^{i}, m^{i}\right)$ are $F_{\tau_{i}}$-measurable random variables, and at time $\tau_{i}$ we buy $l^{i}$ assets paying for them $l^{i} \bar{s}_{\tau_{i}}$ and sell $m^{i}$ assets increasing bank account by $m^{i} \underline{s}_{\tau_{i}}$. Given the initial bank and asset position $(x, y) \in R_{+}^{2}$ and the trading strategy $\left(\tau_{i},\left(l^{i}, m^{i}\right)\right)$, the pair $\left(x_{t}, y_{t}\right)$ is piecewise constant and is of the form: $\left(x_{0}, y_{0}\right)=(x, y),\left(x_{\tau_{1}}, y_{\tau_{1}}\right)=\left(x+m^{1} \underline{s}_{\tau_{1}}-l^{1} \bar{s}_{\tau_{1}}, y-m^{1}+l^{1}\right)$, for $i=1,2, \ldots, r-1$

$$
\left(x_{\tau_{i+1}}, y_{\tau_{i+1}}\right)=\left(x_{\tau_{i}}+m^{i+1} \underline{s}_{\tau_{i+1}}-l^{1} \bar{s}_{\tau_{i+1}}, y_{\tau_{i}}-m^{i+1}+l^{i+1}\right),
$$

and finally $\left(x_{T}, y_{T}\right)=\left(x_{\tau_{r}}, y_{\tau_{r}}\right)$. Since we are not allowed to have short selling, we shall assume that $\left(l^{i}, m^{i}\right) \in \mathcal{A}_{\tau_{i}}\left(x, y, \underline{s}_{\tau_{i}}, \bar{s}_{\tau_{i}}\right)$. Our reward functional is of the form

$$
J_{x, y}\left(\left(\tau_{i},\left(l^{i}, m^{i}\right)\right)\right)=E\left[U\left(x_{T}+y_{T} \underline{s}_{T}\right)\right],
$$

where in $J_{x, y}$ we point out that we start with initial bank account $x$ and have initially $y$ assets. Assuming that the Bellman system ([1.2) has solutions with certain properties, we expect to show that $V_{r}(x, y, 0)$ corresponds to the optimal value of the functional $J_{x, y}$. In the sequel we shall assume the following: 
Assumption (A1). For each $(x, y) \in R_{+}^{2}, n=1, \ldots, r$ and $t \in[0, T]$ we have

$$
E\left[\sup _{t \in[0, T] \cap Q} \bar{V}_{n}\left(x, y, \underline{s}_{t}, \bar{s}_{t}, t\right)\right]<\infty,
$$

where by $Q$ we denote the set of rational numbers.

Notice that because of monotonicity of $U$ and the fact that $(x, y) \in R_{+}^{2}$ we see that the left-hand side of (ㄴ.4) is minorized by $U(x)$, which corresponds to absence of transactions.

The main result of the paper can be formulated as follows:

THEOREM 1.1. Under (A1) there exists $N \subset \Omega$ such that $P(N)=0$ and for $\omega \in \Omega \backslash N$ there are versions of random functions $\bar{V}_{n}\left(x, y, \underline{s}_{t}, \bar{s}_{t}, t\right)$ and $V_{n}(x, y, t)$ for $n \in\{1, \ldots, r\}$, being continuous in $(x, y) \in R_{+}^{2}$, uniformly in $t \in[0, T]$ for $(x, y)$ from compact sets, which means that whenever $\left(x_{k}, y_{k}\right) \rightarrow(x, y)$ as $k \rightarrow$ $\infty$ we have $\lim _{k \rightarrow \infty} \sup _{t \in[0, T]}\left|\bar{V}_{n}\left(x_{k}, y_{k}, \underline{s}_{t}, \bar{s}_{t}, t\right)-\bar{V}_{n}\left(x, y, \underline{s}_{t}, \bar{s}_{t}, t\right)\right|=0$ and $\lim _{k \rightarrow \infty} \sup _{t \in[0, T]}\left|V\left(x_{k}, y_{k}, t\right)-V(x, y, t)\right|=0$. Furthermore, $\bar{V}_{n}\left(x, y, \underline{s}_{t}, \bar{s}_{t}, t\right)$ and $V_{n}(x, y, t)$, for $n \in\{1, \ldots, r\}$, for each $(x, y) \in R_{+}^{2}$ are càdlàg in $t \in[0, T]$ and are such that the system of equations (․2) is satisfied P-a.e.

The proof shall consist of several steps. We need a number of auxiliary results. Those which concern random functions are formulated in Section $\square$ and at the beginning of Section [3. The auxiliary results which concern deterministic functions are shown in Section [5. The proof of the main theorem is in Section [3. Applications of this analytical result are formulated in Section 1 .

\section{AUXILIARY RESULTS}

We start with a lemma in which we shall approximate processes depending on parameters $(x, y)$ by processes with deterministically separated parameters from the random time variables. We have

LEMMA 2.1. Assume that for a given $\omega \in \Omega$ the mapping $R_{+}^{2} \ni(x, y) \mapsto$ $\bar{V}(x, y, t)(\omega)$ is continuous uniformly in $t \in[0, T]$ for $(x, y)$ from compact subsets of $R_{+}^{2}$. Then for any compact set $K \subset R_{+}^{2}$ there is a sequence of pairs $\left(x_{i}, y_{i}\right) \in$ $R_{+}^{2}, i=1,2, \ldots$, and for each $m=1,2, \ldots$ there is a finite sequence of continuous functions $R_{+}^{2} \ni(x, y) \mapsto \phi_{i, m}(x, y)$ for $i=1,2, \ldots, n(m)$ with compact supports taking values in $[0,1]$ such that $\sum_{i=1}^{n(m)} \phi_{i, m}(x, y)=1$ for $(x, y) \in K$, and for the random function $\bar{V}^{m}(x, y, t)(\omega)=\sum_{i=1}^{n(m)} \bar{V}\left(x_{i}, y_{i}, t\right)(\omega) \phi_{i, m}(x, y)$ we have

$$
\sup _{(x, y) \in K} \sup _{t \in[0, T]}\left|\bar{V}^{m}(x, y, t)(\omega)-\bar{V}(x, y, t)(\omega)\right| \rightarrow 0
$$

as $m \rightarrow \infty$. 
Proo f. Denote by $B(x, y, \rho)$ the closed ball in $R^{2}$ with center in $(x, y)$ and radius $\rho>0$, and let $\operatorname{dist}(x, y, B)$ be a distance from $(x, y)$ to $B \subset R^{2}$. For a given compact set $K \subset R_{+}^{2}$ consider a $\rho$ cover of $K$, i.e. a sequence of rational pairs $\left(x_{i}, y_{i}\right)$ with $i=1,2, \ldots, n(1)$ such that $K \subset \bigcup_{i=1}^{n(1)} B\left(x_{i}, y_{i}, \rho\right)$, and then extend this sequence to pairs $\left(x_{i}, y_{i}\right)$ with $i=1,2, \ldots, n(2)$ such that

$$
K \subset \bigcup_{i=1}^{n(2)} B\left(x_{i}, y_{i}, 2^{-1} \rho\right)
$$

We continue the procedure inductively, i.e. having chosen a sequence $\left(x_{i}, y_{i}\right)$ for $i=1,2, \ldots, n(m)$ such that $K \subset \bigcup_{i=1}^{n(m)} B\left(x_{i}, y_{i}, 2^{-m+1} \rho\right)$ we extend the sequence to $\left(x_{i}, y_{i}\right)$ for $i=1,2, \ldots, n(m+1)$ such that $K \subset \bigcup_{i=1}^{n(m+1)} B\left(x_{i}, y_{i}, 2^{-m} \rho\right)$.

Define, for $i \leqslant n(m), g_{i, m}(x, y)=\left(1-\rho^{-1} \operatorname{dist}\left(x, y, B\left(x_{i}, y_{i}, 2^{-m+1} \rho\right)\right)\right)^{+}$ and let

$$
\phi_{i, m}(x, y)=\frac{g_{i, m}(x, y)}{\sum_{j=1}^{n(m)} g_{j, m}(x, y)} .
$$

Clearly, $\sum_{i=1}^{n(m)} \phi_{i, m}(x, y)=1$ for $(x, y) \in K$, and $\phi_{i, m}(x, y)=0$ whenever $(x, y)$ $\notin B\left(x_{i}, y_{i}, 2^{-m+2} \rho\right)$.

Let $\bar{V}^{m}(x, y, t)=\sum_{i=1}^{n(m)} \bar{V}\left(x_{i}, y_{i}, t\right) \phi_{i, m}(x, y)$. Then

$$
\begin{array}{r}
\sup _{(x, y) \in K} \sup _{t \in[0, T]}\left|\sum_{i=1}^{n(m)} \bar{V}\left(x_{i}, y_{i}, t\right) \phi_{i, m}(x, y)-\bar{V}(x, y, t)\right| \\
\leqslant \sup _{(x, y) \in K} \sup _{t \in[0, T]} \sum_{i=1}^{n(m)}\left|\bar{V}\left(x_{i}, y_{i}, t\right)-\bar{V}(x, y, t)\right| \phi_{i, m}(x, y) \rightarrow 0
\end{array}
$$

$P$-a.e. by uniform continuity in $t$ of $\bar{V}$ with respect to $(x, y) \in K$.

Using Lemma 2.1 we are going now to show that nice properties of the state process are preserved by the Snell envelope taken over a finite time horizon.

Proposition 2.1. Assume that there is $N \subset \Omega, P(N)=0$ such that, for $\omega \in \Omega \backslash N$ and $(x, y) \in R_{+}^{2}, \bar{V}(x, y, t)(\omega)$ is a càdlàg process adapted to $\left(F_{t}\right)$ such that $(x, y) \mapsto \bar{V}(x, y, t)$ is continuous uniformly in $t$ for $(x, y)$ from compact subsets of $R_{+}^{2}$, and $E\left[\sup _{t \in[0, T]}|\bar{V}(x, y, t)|\right]<\infty$. Then, for $\omega \in \Omega \backslash \bar{N}, P(\bar{N})=0$, there is a càdlàg process $(V(x, y, t))(\omega)$, adapted to $\left(F_{t}\right)$, such that $(x, y) \mapsto$ $V(x, y, t)$ is continuous uniformly in $t$ for $(x, y)$ from compact subsets of $R_{+}^{2}$, and

$$
V(x, y, t)=\operatorname{ess}_{t \leqslant \tau \leqslant T} E\left[\bar{V}(x, y, \tau) \mid F_{t}\right] P \text {-a.e. }
$$

Proof. Let $\bar{V}^{m}(x, y, t)$ be a sequence of the form

$$
\bar{V}^{m}(x, y, t)(\omega)=\sum_{i=1}^{n(m)} \bar{V}\left(x_{i}, y_{i}, t\right)(\omega) \phi_{i, m}(x, y)
$$


for which (2.II) holds. Let

$$
V^{m}(x, y, t):=\operatorname{ess~sup}_{t \leqslant \tau \leqslant T} \sum_{i=1}^{n(m)} E\left[\bar{V}\left(x_{i}, y_{i}, \tau\right) \mid F_{t}\right] \phi_{i, m}(x, y) .
$$

By Theorems 2 and 3 of [ [ 7$]$ as well as Theorem 2.46 of [8] there is $N_{1}, P\left(N_{1}\right)=0$ such that for $\omega \in \Omega \backslash N_{1}$ and $(x, y) \in Q_{+}^{2}$ the mapping $[0, T] \ni t \mapsto V^{m}(x, y, t)$ is càdlàg. Furthermore, there is $N_{2} \subset \Omega, N_{1} \subset N_{2}$, and $P\left(N_{2}\right)=0$ such that for $(x, y),\left(x^{\prime}, y^{\prime}\right) \in Q_{+}^{2}$ and $t \in[0, T]$ such that

$$
\begin{aligned}
\mid \bar{V}^{m}(x, y, t) & -\bar{V}^{m}\left(x^{\prime}, y^{\prime}, t\right) \mid \\
& \leqslant \sum_{i=1}^{n(m)} E\left[\sup _{u}\left|\bar{V}\left(x_{i}, y_{i}, u\right)\right| \mid F_{t}\right]\left|\phi_{i, m}(x, y)-\phi_{i, m}\left(x^{\prime}, y^{\prime}\right)\right|
\end{aligned}
$$

and for each $i=1,2, \ldots$, the mapping $[0, T] \ni t \mapsto E\left[\sup _{u} \mid \bar{V}\left(x_{i}, y_{i}, u\right) \| F_{t}\right]$ is càdlàg (by Theorem 2.46 of [8] again). Consequently, using ([2.3) we may define $V^{m}(x, y, t)$ for $(x, y) \in R_{+}^{2} \backslash Q_{+}^{2}$ as a unique limit, independent of a chosen sequence of $V^{m}\left(x^{n}, y^{n}, t\right)$, where $Q_{+}^{2} \ni\left(x_{n}, y_{n}\right) \rightarrow(x, y)$. By continuity, ([2.3]) is also satisfied for $(x, y),\left(x^{\prime}, y^{\prime}\right) \in R_{+}^{2}$. Furthermore, from (2.3) we also infer that for $\omega \in \Omega \backslash N_{2}$ the mapping $(x, y) \mapsto V^{m}(x, y, t)$ is continuous uniformly in $t \in[0, T]$ for $(x, y)$ from compact sets.

In addition, for $(x, y) \in R_{+}^{2}$ the mapping $[0, T] \ni t \mapsto V^{m}(x, y, t)$ is càdlàg. In fact, this holds for $(x, y) \in Q_{+}^{2}$. If $t_{k} \downarrow t$ and $Q_{+}^{2} \ni\left(x_{n}, y_{n}\right) \rightarrow(x, y) \in R_{+}^{2} \backslash Q_{+}^{2}$ as $k \rightarrow \infty, n \rightarrow \infty$, we have

$$
\begin{aligned}
& \left|V^{m}\left(x, y, t_{k}\right)-V^{m}(x, y, t)\right| \\
\leqslant & \left|V^{m}\left(x, y, t_{k}\right)-V^{m}\left(x_{n}, y_{n}, t_{k}\right)\right| \\
& +\left|V^{m}\left(x_{n}, y_{n}, t_{k}\right)-V^{m}\left(x_{n}, y_{n}, t\right)\right|+\left|V^{m}\left(x_{n}, y_{n}, t\right)-V^{m}(x, y, t)\right| \\
\leqslant & \sum_{i=1}^{n(m)} E\left[\sup _{u}\left|\bar{V}\left(x_{i}, y_{i}, u\right)\right| \mid F_{t_{k}}\right]\left|\phi_{i, m}(x, y)-\phi_{i, m}\left(x_{n}, y_{n}\right)\right| \\
& +\left|V^{m}\left(x_{n}, y_{n}, t_{k}\right)-V^{m}\left(x_{n}, y_{n}, t\right)\right| \\
& \left.+\sum_{i=1}^{n(m)} \underset{u}{\operatorname{mou}}\left|\bar{V}\left(x_{i}, y_{i}, u\right)\right| \mid F_{t}\right]\left|\phi_{i, m}\left(x_{n}, y_{n}\right)-\phi_{i, m}(x, y)\right| \\
= & : a_{k}(n)+b_{k}(n)+c(n) .
\end{aligned}
$$

For $\epsilon>0$ there is $n(\epsilon)$ such that for $n \geqslant n(\epsilon)$ we have $a_{k}(n) \leqslant \frac{\epsilon}{3}$ for any $k$ and $c(n) \leqslant \frac{\epsilon}{3}$. For fixed $n \geqslant n(\epsilon)$ we can find $k$ such that $b_{k}(n) \leqslant \frac{\epsilon}{3}$. Since $\epsilon$ can be chosen arbitrarily small, we have $V^{m}\left(x, y, t_{k}\right) \rightarrow V^{m}(x, y, t)$ as $k \rightarrow \infty$. The existence of the left limit of the mapping $[0, T] \ni t \mapsto V^{m}(x, y, t)$ for $(x, y) \in$ 
$R_{+}^{2} \backslash Q_{+}^{2}$ can be proved in a similar way. Consequently, for $\omega \in \Omega \backslash N_{2}$ we infer that $V^{m}(x, y, t)$ is continuous in $(x, y) \in R_{+}^{2}$ uniformly in $t$ for $(x, y)$ for compact sets, and the mapping $[0, T] \ni t \mapsto V^{m}(x, y, t)$ is càdlàg. Using Theorems 2 and 3 of [7] as well as Theorem 2.46 of [8], we see that there is $N_{3} \subset \Omega$ such that $N_{2} \subset N_{3}, P\left(N_{3}\right)=0$ and for $\omega \in \Omega \backslash N_{3},(x, y) \in Q_{+}^{2}$ there is a càdlàg version of the supermartingale

$$
V(x, y, t)=\operatorname{essip}_{t \leqslant \tau \leqslant T} E\left[\bar{V}(x, y, \tau) \mid F_{t}\right],
$$

and for balls $K=B(0, n), n=1,2, \ldots$, we have

$$
\begin{aligned}
& \text { (2.5) } \sup _{t \in[0, T] \cap Q} \sup _{(x, y) \in Q_{+}^{2} \cap K}\left|V(x, y, t)-V^{m}(x, y, T)\right| \\
& \leqslant \sup _{t \in[0, T] \cap Q} \sup _{(x, y) \in Q_{+}^{2} \cap K} \sum_{i=1}^{n(m)} E\left[\sup _{u \in[0, T]} \mid \bar{V}\left(x_{i}, y_{i}, u\right)-\bar{V}(x, y, u) \| F_{t}\right] \phi_{i, m}(x, y)
\end{aligned}
$$

which by Lemma 2.$]$ tends to zero $P$-a.e. The claim now follows from Lemma 5.2 below.

\section{PROOF OF THE MAIN RESULT}

Before we prove the main result we need a regularity property of the first step in the Bellman system, i.e. regularity (in the sense defined below) of the process $\tilde{V}_{1}$ defined as

$$
\tilde{V}_{1}(x, y, t):=E\left[V_{0}\left(x, y, \underline{s}_{T}, \bar{s}_{T}\right) \mid F_{t}\right] .
$$

We have the following

Proposition 3.1. There is a version of $\tilde{V}_{1}$ and $N \subset \Omega$ such that $P(N)=0$ and for $\omega \in \Omega \backslash N$ and $(x, y) \in R_{+}^{2}$ the mapping $[0, T] \ni t \mapsto \tilde{V}_{1}(x, y, t)(\omega)$ is càdlàg, and for any compact set $K \subset R_{+}^{2}$ the mapping $K \ni(x, y) \mapsto \tilde{V}_{1}(x, y, t)(\omega)$ is continuous uniformly in $t \in[0, T]$.

Pro of. The proof consists of two steps.

S te p 1 . We claim that for $M \in Q_{+}$there is a version of

$$
\tilde{V}_{1}^{M}(x, y, t):=E\left[V_{0}\left(x, y, \underline{s}_{T} \wedge M, \bar{s}_{T} \wedge M\right) \mid F_{t}\right]
$$

such that for $M \in Q_{+}$there is $N \subset \Omega, P(N)=0$ such that for $\omega \in \Omega \backslash N$ the mapping $[0, T] \ni t \mapsto \tilde{V}_{1}^{M}(x, y, t)$ is càdlàg, and for any compact set $K \subset R_{+}^{2}$ the mapping $K \ni(x, y) \mapsto \tilde{V}_{1}^{M}(x, y, t)$ is continuous uniformly in $t \in[0, T]$. 
For fixed $M \in Q_{+}$, a compact set $K$ and $\varepsilon>0$, by the Stone-Weierstrass theorem (Theorem 13.4 of [110]) there are sequences of continuous functions $\phi_{1, i}^{\varepsilon}, \phi_{2, i}^{\varepsilon}, i=1,2, \ldots, k(\varepsilon)$, such that for $\omega \in \Omega$

$$
\sup _{(x, y) \in K}\left|V_{0}\left(x, y, \underline{s}_{T} \wedge M, \bar{s}_{T} \wedge M\right)-\sum_{i=1}^{k(\varepsilon)} \phi_{1, i}^{\varepsilon}(x, y) \phi_{2, i}^{\varepsilon}\left(\underline{s}_{T} \wedge M, \bar{s}_{T} \wedge M\right)\right| \leqslant \varepsilon .
$$

By Proposition 3 of [15] there is a measurable function on $R_{+}^{2} \times R \times \Omega$ such that its value $\tilde{V}_{1}^{M}(x, y, t)$ for each $(x, y) \in R_{+}^{2}$ is indistinguishable from an optional process. By Theorem V.20 in [4] this process is indistinguishable from the càdlàg process. Therefore, due to Lemma 1 of [15] (see also Lemma 5 of [6]) there is $N_{1} \subset \Omega, P\left(N_{1}\right)=0$ such that for $\omega \in \Omega \backslash N_{1}$ and $(x, y) \in R_{+}^{2}$ the trajectories $t \mapsto \tilde{V}_{1}^{M}$ are càdlàg. Let $\varepsilon_{n} \downarrow 0$. By Theorems 2.44 and 2.46 of [8] there is $N, N_{1} \subset N, P(N)=0$ such that, for each $n=1,2, \ldots$, the processes $t \rightarrow E\left[\phi_{2}^{\varepsilon_{n}}\left(\underline{s}_{T} \wedge M, \bar{s}_{T} \wedge M\right) \mid F_{t}\right]$ are càdlàg martingales. Let

$$
\hat{V}_{1}^{M, n}(x, y, t):=\sum_{i=1}^{k\left(\varepsilon_{n}\right)} \phi_{1}^{\varepsilon_{n}}(x, y) E\left[\phi_{2}^{\varepsilon_{n}}\left(\underline{s}_{T} \wedge M, \bar{s}_{T} \wedge M\right) \mid F_{t}\right] .
$$

Then, for $\omega \in \Omega \backslash N_{2}, \hat{V}_{1}^{M, n}(x, y, t)$ is a càdlàg martingale, continuous in $(x, y) \in$ $K$, uniformly in $t \in[0, T]$.

We are going now to show that

$$
\sup _{t \in[0, T]} \sup _{(x, y) \in K \cap Q_{+}^{2}}\left|\tilde{V}_{1}^{M}(x, y, t)-\hat{V}_{1}^{M, n}(x, y, t)\right| \rightarrow 0
$$

in probability. By Doob's inequality for submartingales (see Theorem V.24 of [5]), for $p>1$

$$
\begin{aligned}
& E\left\{\sup _{t \in[0, T]} \sup _{(x, y) \in K \cap Q_{+}^{2}}\left|\tilde{V}_{1}^{M}(x, y, t)-\hat{V}_{1}^{M, n}(x, y, t)\right|^{p}\right\} \\
\leqslant & C E\left\{\sup _{(x, y) \in K}\left|V_{0}\left(x, y, \underline{s}_{T} \wedge M, \bar{s}_{T} \wedge M\right)-\sum_{i=1}^{k\left(\varepsilon_{n}\right)} \phi_{1}^{\varepsilon_{n}}(x, y) \phi_{2}^{\varepsilon_{n}}\left(\underline{s}_{T} \wedge M, \bar{s}_{T} \wedge M\right)\right|^{p}\right\} \\
\leqslant & C \varepsilon_{n}^{p} .
\end{aligned}
$$

Consequently, for a suitably chosen subsequence $n_{r}$ we have

$$
\sup _{t \in[0, T]} \sup _{(x, y) \in K \cap Q_{+}^{2}}\left|\tilde{V}_{1}^{M}(x, y, t)-\hat{V}_{1}^{M, n_{r}}(x, y, t)\right| \rightarrow 0
$$

as $r \rightarrow \infty, P$-a.e. Using Lemma 5.2, we obtain the desired properties of $\tilde{V}_{1}^{M}(x, y, t)$. 
S te p 2. By Proposition 3 of [15], then Theorem V.20 of [4] and Lemma 1 of [15], there is a version of $\tilde{V}_{1}(x, y, t)$ such that for $\omega \in \Omega \backslash \tilde{N}$, where $P(\tilde{N})=0$, for $(x, y) \in R_{+}^{2}$ the trajectories $t \rightarrow \tilde{V}_{1}(x, y, t)$ are càdlàg. By Step 1 , for $Q_{+} \ni$ $M_{n} \rightarrow \infty$ there is $N^{\prime}$ such that $\tilde{N} \subset N^{\prime}, P\left(N^{\prime}\right)=0$ such that, for $\omega \in \Omega \backslash N^{\prime}$, $(x, y) \in Q_{+}^{2}$, the trajectories $[0, T] \ni t \mapsto \tilde{V}_{1}^{M_{n}}(x, y, t)$ are càdlàg and $K \ni(x, y)$ $\mapsto \tilde{V}_{1}^{M_{n}}(x, y, t)$ is continuous uniformly in $t$. By Doob's inequality for submartingales (Theorem V.24 of [5]), for $p>1$

$$
\begin{aligned}
& E\left\{\sup _{t \in[0, T]} \sup _{(x, y) \in K \cap Q_{+}^{2}}\left|\tilde{V}_{1}(x, y, t)-\hat{V}_{1}^{M_{n}}(x, y, t)\right|^{p}\right\} \\
& \quad \leqslant C E\left\{\sup _{x, y \in K}\left|V_{1}\left(x, y, \underline{s}_{T}, \bar{s}_{T}\right)-V_{1}\left(x, y, \underline{s}_{T} \wedge M, \bar{s}_{T} \wedge M\right)\right|^{p}\right\} \rightarrow 0
\end{aligned}
$$

as $n \rightarrow \infty$. Therefore, we have convergence in probability and for a suitably chosen subsequence $\left(n_{r}\right)$ the convergence

$$
\sup _{t \in[0, T]} \sup _{(x, y) \in K \cap Q_{+}^{2}}\left|\tilde{V}_{1}(x, y, t)-\hat{V}_{1}^{M_{n_{r}}}(x, y, t)\right| \rightarrow 0
$$

$P$-a.e. as $r \rightarrow \infty$. It remains now to use Lemma 5.2 to complete the proof.

Remark 3.1. Notice that in both Steps 1 and 2 we used Proposition 3 of [15], and next Theorem V.20 of [4] and Lemma 1 of [15] to get, for each $(x, y) \in R_{+}^{2}$, càdlàg versions of the processes $\tilde{V}_{1}^{M}$ or $\tilde{V}_{1}$. In view of Lemma 5.2 we need only càdlàg versions of the processes $\hat{V}_{1}^{n, M}$ and then $\hat{V}_{1}^{M_{n}}$, since by uniform convergence for $(x, y) \in Q_{+}^{2}$ and $t \in[0, T]$ we may define (in a unique way) the processes $\tilde{V}_{1}^{M}$ and $\tilde{V}_{1}$ to be càdlàg. In Proposition 2.$]$ we had no such possibility and we had to use a direct (not simplified) version of Lemma 5.2

We are now in a position to complete the proof of the main theorem.

Pr o of of Th e or e m ㄷ.]. By Proposition B.], $\tilde{V}_{1}(x, y, t)$ is continuous in $(x, y)$ from compact subsets of $R_{+}^{2}$ uniformly in $t \in[0, T]$ and is càdlàg in $t$ for fixed $(x, y) \in R_{+}^{2}$. Using Lemma $\mathbf{5 . 3}$, we see that $\bar{V}_{1}\left(x, y, \underline{s}_{t}, \bar{s}_{t}, t\right)$ is continuous in $(x, y)$ from compact subsets of $R_{+}^{2}$ uniformly in $t \in[0, T]$ and is càdlàg in $t$ for fixed $(x, y) \in R_{+}^{2}$. By Proposition 2.$]$ we infer that $V_{1}(x, y, t)$ is continuous in $(x, y)$ from compact subsets of $R_{+}^{2}$ uniformly in $t \in[0, T]$ and is càdlàg in $t$ for fixed $(x, y) \in R_{+}^{2}$. We now proceed by induction. Assume that $V_{n}(x, y, t)$ is continuous in $(x, y)$ from compact subsets of $R_{+}^{2}$ uniformly in $t \in[0, T]$ and is càdlàg in $t$ for fixed $(x, y) \in R_{+}^{2}$. Then, by Lemma [5.3, $\bar{V}_{n+1}\left(x, y, \underline{s}_{t}, \bar{s}_{t}, t\right)$ is continuous in $(x, y)$ from compact subsets of $R_{+}^{2}$ uniformly in $t \in[0, T]$ and is càdlàg in $t$ for fixed $(x, y) \in R_{+}^{2}$. Using Proposition 2.1 again, we infer that $V_{n+1}(x, y, t)$ is continuous in $(x, y)$ from compact subsets of $R_{+}^{2}$ uniformly in $t \in[0, T]$ and is càdlàg in $t$ for fixed $(x, y) \in R_{+}^{2}$. This completes the proof. 


\section{APPLICATIONS}

In this section we shall formulate some implications of the analytical result formulated in Theorem ㄴ.1.

Proposition 4.1. Under (A1) we have

$$
\sup _{\left(\tau_{i},\left(l^{i}, m^{i}\right)\right)} J_{x, y}\left(\left(\tau_{i},\left(l^{i}, m^{i}\right)\right)\right)=V_{r}(x, y, 0) .
$$

Furthermore, for a given $(x, y) \in R_{+}^{2}$ and $\varepsilon>0$ there is an $\varepsilon$-optimal investment strategy $\left(\tau_{i},\left(l^{i}, m^{i}\right)\right)$ given by

$$
\begin{aligned}
& \tau_{1}:=\inf \left\{t \geqslant 0: V_{r}(x, y, t) \leqslant \bar{V}_{r}\left(x, y, \underline{s}_{t}, \bar{s}_{t}, t\right)+\varepsilon / r\right\}, \\
& \left(l^{1}, m^{1}\right):=\left(\hat{l}^{1}, \hat{m}^{1}\right) \in \mathcal{A}_{\tau_{1}}\left(x, y, \underline{s}_{\tau_{1}}, \bar{s}_{\tau_{1}}\right), \\
& \bar{V}_{r}\left(x, y, \underline{s}_{\tau_{1}}, \bar{s}_{\tau_{1}}, \tau_{1}\right)=V_{r-1}\left(x+\hat{m}^{1} \underline{s}_{\tau_{1}}-\hat{l}^{1} \bar{s}_{\tau_{1}}, y-\hat{m}^{1}+\hat{l}^{1}, \tau_{1}\right), \\
& \tau_{i+1}:=\inf \left\{t \geqslant \tau_{i}: V_{r-i}\left(x_{\tau_{i}}, y_{\tau_{i}}, t\right) \leqslant \bar{V}_{r-i}\left(x_{\tau_{i}}, y_{\tau_{i}}, \underline{s}_{t}, \bar{s}_{t}, t\right)+\varepsilon / r\right\}, \\
& \left(l^{i+1}, m^{i+1}\right):=\left(\hat{l}^{i+1}, \hat{m}^{i+1}\right) \in \mathcal{A}_{\tau_{i+1}}\left(x_{\tau_{i}}, y_{\tau_{i}}, \underline{s}_{\tau_{i+1}}, \bar{s}_{\tau_{i+1}}\right), \\
& \bar{V}_{r-i}\left(x_{\tau_{i}}, y_{\tau_{i}}, \underline{s}_{\tau_{i+1}}, \bar{s}_{\tau_{i+1}}, \tau_{i+1}\right) \\
& =V_{r-i-1}\left(x_{\tau_{i}}+\hat{m}^{i+1} \underline{s}_{\tau_{i+1}}-\hat{l}^{i+1} \bar{s}_{\tau_{i+1}}, y_{\tau_{i}}-\hat{m}^{i+1}+\hat{l}^{i+1}, \tau_{i+1}\right), \\
& V_{0}\left(x_{\tau_{r}}, y_{\tau_{r}}, \underline{s}_{T}, \bar{s}_{T}, T\right)=U\left(x+y_{\tau_{r}} \underline{s}_{T}\right),
\end{aligned}
$$

where for $i=0,1, \ldots, r-1, x_{\tau_{i+1}}=x_{\tau_{i}}+\hat{m}^{i+1} \underline{s}_{\tau_{i+1}}-\hat{l}^{i+1} \bar{s}_{\tau_{i+1}}$ and $y_{\tau_{i+1}}=$ $y_{\tau_{i}}-\hat{m}^{i+1}+\hat{l}^{i+1}$.

Proof. Consider an arbitrary investment strategy $\left(\tau_{i},\left(l^{i}, m^{i}\right)\right)$ and corresponding market positions $\left(x_{t}, y_{t}\right)$ with $\left(x_{0}, y_{0}\right)=(x, y)$. Then the value of the reward functional $J_{x, y}$ is given by $E\left[V_{0}\left(x_{T}, y_{T}, \bar{s}_{T}, \underline{s}_{T}\right)\right]$, and

$$
\begin{aligned}
\bar{V}_{1}\left(x_{\tau_{r}}, y_{\tau_{r}}, \underline{s}_{\tau_{r}}, \bar{s}_{\tau_{r}}, \tau_{r}\right) \\
\quad \geqslant E\left[V_{0}\left(x_{\tau_{r}}+m^{r} \underline{s}_{\tau_{r}}-l^{r} \bar{s}_{t}, y_{\tau_{r}}-m^{r}+l^{r}, \underline{s}_{T}, \bar{s}_{T}\right) \mid F_{\tau_{r}}\right] .
\end{aligned}
$$

Moreover,

$$
V_{1}\left(x_{\tau_{r-1}}, y_{\tau_{r-1}}, \tau_{r-1}\right) \geqslant E\left[\bar{V}_{1}\left(x_{\tau_{r}}, y_{\tau_{r}}, \underline{s}_{\tau_{r}}, \bar{s}_{\tau_{r}}, \tau_{r}\right) \mid F_{\tau_{r-1}}\right]
$$

and for $i=0,1, \ldots, r-1$

$$
\begin{aligned}
& \bar{V}_{r-i}\left(x_{\tau_{i}}, y_{\tau_{i}}, \underline{s}_{\tau_{i+1}}, \bar{s}_{\tau_{i+1}}, \tau_{i+1}\right) \\
& \quad \geqslant V_{r-i-1}\left(x_{\tau_{i}}+m^{i+1} \underline{s}_{\tau_{i+1}}-l^{i+1} \bar{s}_{\tau_{i+1}}, y_{\tau_{i}}-m^{i+1}+l^{i+1}, \tau_{i+1}\right),
\end{aligned}
$$




$$
V_{r-i}\left(x_{\tau_{i}}, y_{\tau_{i}}, \tau_{i+1}\right) \geqslant E\left[\bar{V}_{r-i}\left(x_{\tau_{i}}, y_{\tau_{i}}, \underline{s}_{\tau_{i+1}}, \bar{s}_{\tau_{i+1}}, \tau_{i+1}\right) \mid F_{\tau_{i}}\right] .
$$

For the strategy defined in (4.2) we have equalities in (4.3) and (4.5) (because the strategy maximizes the left-hand sides of (4.3) and (4.5)), while in (4.4) and (4.6) inverse inequalities with $V_{1}$ or $V_{r-i}$ decreased by $\frac{\varepsilon}{r}$. Now we can condition the final reward using (4.3)-(4.6) and obtain

$$
\begin{aligned}
& E\left[V_{0}\left(x_{T}, y_{T}, \bar{s}_{T}, \underline{s}_{T}\right)\right] \\
& \quad \leqslant E\left[\bar{V}_{1}\left(x_{\tau_{r}}, y_{\tau_{r}}, \underline{s}_{\tau_{r}}, \bar{s}_{\tau_{r}}, \tau_{r}\right)\right] \leqslant E\left[V_{1}\left(x_{\tau_{r-1}}, y_{\tau_{r-1}}, \tau_{r-1}\right)\right] \leqslant \ldots \\
& \leqslant E\left[\bar{V}_{r-i}\left(x_{\tau_{i}}, y_{\tau_{i}}, \underline{s}_{\tau_{i+1}}, \bar{s}_{\tau_{i+1}}, \tau_{i+1}\right)\right] \leqslant E\left[V_{r-i}\left(x_{\tau_{i}}, y_{\tau_{i}}, \tau_{i+1}\right)\right] \leqslant \ldots \\
& \leqslant E\left[\bar{V}_{r}\left(x_{\tau_{1}}, y_{\tau_{1}}, \underline{s}_{\tau_{1}}, \bar{s}_{\tau_{1}}, \tau_{1}\right)\right] \leqslant V_{r}(x, y, 0) .
\end{aligned}
$$

Notice that for the strategy (4.2) we obtain in (4.7)

$$
E\left[V_{0}\left(x_{T}, y_{T}, \bar{s}_{T}, \underline{s}_{T}\right)\right] \geqslant V_{r}(x, y, 0)-\varepsilon,
$$

which is the desired $\varepsilon$-optimality.

In Proposition 4. 1 above we had only $\varepsilon$-optimality of strategies, since the existence of optimal stopping times in (ㅍ.2) could not be guaranteed. Under additional assumptions we can however expect to get optimal strategies. Assume the following:

Assumption (A2). The filtration $\left(F_{t}\right)$ is quasi-left continuous (see Definition 3.33 in [ [8]]), and the processes $\left(\underline{s}_{t}\right)$ and $\left(\bar{s}_{t}\right)$ are quasi-left continuous (see Definition 4.22 in [8] ).

PROPOSITION 4.2. Under the assumptions (A1) and (A2) there is an optimal strategy $\left(\tau_{i},\left(l^{i}, m^{i}\right)\right)$ maximizing (ㄸ.3). It is of the form

$$
\begin{aligned}
& \tau_{1}:=\inf \left\{t \geqslant 0: V_{r}(x, y, t)=\bar{V}_{r}\left(x, y, \underline{s}_{t}, \bar{s}_{t}, t\right)\right\}, \\
& \left(l^{1}, m^{1}\right):=\left(\hat{l}^{1}, \hat{m}^{1}\right) \in \mathcal{A}_{\tau_{1}}\left(x, y, \underline{s}_{\tau_{1}}, \bar{s}_{\tau_{1}}\right), \\
& \bar{V}_{r}\left(x, y, \underline{s}_{\tau_{1}}, \bar{s}_{\tau_{1}}, \tau_{1}\right)=V_{r-1}\left(x+\hat{m}^{1} \underline{s}_{\tau_{1}}-\hat{l}^{1} \bar{s}_{\tau_{1}}, y-\hat{m}^{1}+\hat{l}^{1}, \tau_{1}\right), \\
& \tau_{i+1}:=\inf \left\{t \geqslant \tau_{i}: V_{r-i}\left(x_{\tau_{i}}, y_{\tau_{i}}, t\right)=\bar{V}_{r-i}\left(x_{\tau_{i}}, y_{\tau_{i}}, \underline{s}_{t}, \bar{s}_{t}, t\right)\right\}, \\
& \left(l^{i+1}, m^{i+1}\right):=\left(\hat{l}^{i+1}, \hat{m}^{i+1}\right) \in \mathcal{A}_{\tau_{i+1}}\left(x_{\tau_{i}}, y_{\tau_{i}}, \underline{s}_{\tau_{i+1}}, \bar{s}_{\tau_{i+1}}\right), \\
& \bar{V}_{r-i}\left(x_{\tau_{i}}, y_{\tau_{i}}, \underline{s}_{\tau_{i+1}}, \bar{s}_{\tau_{i+1}}, \tau_{i+1}\right) \\
& =V_{r-i-1}\left(x_{\tau_{i}}+\hat{m}^{i+1} \underline{s}_{\tau_{i+1}}-\hat{l}^{i+1} \bar{s}_{\tau_{i+1}}, y_{\tau_{i}}-\hat{m}^{i+1}+\hat{l}^{i+1}, \tau_{i+1}\right), \\
& V_{0}\left(x_{\tau_{r}}, y_{\tau_{r}}, \underline{s}_{T}, \bar{s}_{T}, T\right)=U\left(x+y_{\tau_{r}} \underline{s}_{T}\right) \\
& \text { with } x_{\tau_{i+1}}=x_{\tau_{i}}+\hat{m}^{i+1} \underline{s}_{\tau_{i+1}}-\hat{l}^{i+1} \bar{s}_{\tau_{i+1}} \text { and } y_{\tau_{i+1}}=y_{\tau_{i}}-\hat{m}^{i+1}+\hat{l}^{i+1} \text {. }
\end{aligned}
$$


P r o o f. By Theorem I.3 of [2] or Theorem I.2.2 of [12] we have only to show that the processes $V_{k}$ and $\bar{V}_{k}$ are quasi-left continuous for $k=0,1, \ldots, r$. Notice first that by quasi-left continuity of $\left(F_{t}\right)$ the process $\tilde{V}_{1}$ defined in (B. I) is quasi-left continuous. Now, if a sequence of stopping times $\sigma_{n} \uparrow \sigma \leqslant T$ as $n \rightarrow \infty$, then by (A2) we have $\underline{s}_{\sigma_{n}} \rightarrow \underline{s}_{\sigma}$ and $\bar{s}_{\sigma_{n}} \rightarrow \bar{s}_{\sigma}, P$-a.e., as $n \rightarrow \infty$. Therefore, by (5.8), using quasi-left continuity of $\tilde{V}_{1}$, we have

$$
\begin{aligned}
& \bar{V}_{1}\left(x, y, \underline{s}_{\sigma_{n}}, \bar{s}_{\sigma_{n}}, \sigma_{n}\right) \rightarrow \sup _{(l, m) \in A\left(x, y, \underline{s}_{\sigma}^{-}, \bar{s}_{\sigma}^{-}\right)} \tilde{V}_{1}^{-}\left(x+m \underline{s}_{\sigma}^{-}-l \bar{s}_{\sigma}^{-}, y-m+l, \sigma\right) \\
= & \sup _{(l, m) \in A\left(x, y, \underline{s}_{\sigma}, \bar{s}_{\sigma}\right)} \tilde{V}_{1}\left(x+m \underline{s}_{\sigma}-l \bar{s}_{\sigma}, y-m+l, \sigma\right)=\bar{V}_{1}\left(x, y, \underline{s}_{\sigma}, \bar{s}_{\sigma}, \sigma\right)
\end{aligned}
$$

$P$-a.e., where $\tilde{V}_{1}^{-}$denotes the left limit. Consequently, $\bar{V}_{1}$ is quasi-left continuous. It remains to show that $V_{1}$ is quasi-left continuous since then the proof can be continued by induction. For this purpose we repeat the arguments of the proof of Theorem I.2.2 of [12] or Theorem I.5 of [2]. Let $\sigma_{n} \uparrow \sigma \leqslant T$ as $n \rightarrow \infty$. Since $V_{1}(x, y, t)$ for fixed $x, y \in R_{+}$is a supermartingale, we infer that $V_{1}\left(x, y, \sigma_{n}\right) \geqslant$ $V_{1}(x, y, \sigma)$ and

$$
E\left[V_{1}\left(x, y, \sigma_{n}\right)\right] \rightarrow E\left[V_{1}^{-}(x, y, \sigma)\right] \geqslant E\left[V_{1}(x, y, \sigma)\right] .
$$

Let $\zeta\left(\sigma_{n}\right)=\inf \left\{t \geqslant \sigma_{n}: V_{1}(x, y, t)=\bar{V}_{1}\left(x, y, \underline{s}_{t}, \bar{s}_{t}, t\right)\right\}$. It is clear that $\left(\zeta\left(\sigma_{n}\right)\right)$ is increasing, as $n \rightarrow \infty$, to a stopping time $\zeta$. Furthermore, by quasi-left continuity of $\bar{V}_{1}$ we have

$$
\begin{aligned}
E\left[V_{1}\left(x, y, \sigma_{n}\right)\right]=E\left[\bar{V}_{1}\left(x, y, \underline{s}_{\zeta\left(\sigma_{n}\right)}, \bar{s}_{\zeta\left(\sigma_{n}\right)}, \zeta\left(\sigma_{n}\right)\right)\right] & \\
& \rightarrow E\left[\bar{V}_{1}\left(x, y, \underline{s}_{\zeta}, \bar{s}_{\zeta}, \zeta\right)\right]
\end{aligned}
$$

and it is clear that $\zeta \geqslant \sigma$. Therefore,

$$
E\left[\bar{V}_{1}\left(x, y, \underline{s}_{\zeta}, \bar{s}_{\zeta}, \zeta\right)\right] \leqslant E\left[V_{1}(x, y, \zeta)\right] \leqslant E\left[V_{1}(x, y, \sigma)\right],
$$

and from (4.9) and (4.10) we obtain $\lim _{n \rightarrow \infty} E\left[V_{1}\left(x, y, \sigma_{n}\right)\right]=E\left[V_{1}(x, y, \sigma)\right]$, which is only possible when $V_{1}^{-}(x, y, \sigma)=V_{1}(x, y, \sigma), P$-a.e., i.e. when $V_{1}$ is quasi-left continuous.

We construct below a family of examples for which the assumption (A2) is satisfied.

EXAMPLE 4.1. Let $\left(B_{t}^{H}\right)$ be a fractional Brownian motion with parameter $H>\frac{1}{2}$ and continuous trajectories. Let $\left(F_{t}\right)$ be a completed filtration generated by $\left(B_{t}^{H}\right)$. It follows from Theorem 3.1 and formula (3.1) of [11] together with Corollaries 2.7.8, 2.7.9 and Problems 7.1, 7.6 of [9] that there is a Brownian motion 
$\left(W_{t}\right)$ such that its completed filtration coincides with $\left(F_{t}\right)$, and therefore $\left(F_{t}\right)$ is continuous and, in particular, quasi-left continuous. Define the processes

$$
\underline{s}_{t}=\underline{s}_{0} \exp \left\{\int_{0}^{t} a_{s} d s+\int_{0}^{t} c_{s} d B_{s}^{H}\right\}
$$

and

$$
\bar{s}_{t}=\bar{s}_{0} \exp \left\{\int_{0}^{t} b_{s} d s+\int_{0}^{t} c_{s} d B_{s}^{H}\right\},
$$

where $c \in L^{2}(0, T)$ and $\left(a_{t}\right),\left(b_{t}\right)$ are $\left(F_{t}\right)$-adapted such that $a_{t}<b_{t}$ for $t \in[0, T]$, and $a, b \in L^{1}[\Omega \times[0, T]]$. Then $\left(\underline{s}_{t}\right)$ and $\left(\bar{s}_{t}\right)$ are continuous, and one can show that the processes $\bar{V}_{k}$ and $V_{k}$ are continuous for $k=0,1, \ldots, r$.

Proposition 4.1$]$ concerned the case when we had not more than $r$ transactions over time interval $[0, T]$. A natural question is what happens when we let $r \rightarrow \infty$. Since the bid price $\underline{s}_{t}$ is strictly smaller than the ask price $\bar{s}_{t}$, we may expect that we shall have a finite number of transactions in time horizon $[0, T]$, although the number of transactions might be random, not necessarily bounded uniformly in $\omega$. Notice that $V_{r}$ and $\bar{V}_{r}$ are nondecreasing in $r$. Consequently, the processes $V(x, y, t):=\lim _{r \rightarrow \infty} V_{r}(x, y, t)$ and $\bar{V}\left(x, y, \underline{s}_{t}, \bar{s}_{t}, t\right):=\lim _{n \rightarrow \infty} \bar{V}_{r}\left(x, y, \underline{s}_{t}, \bar{s}_{t}, t\right)$ are well defined although might be equal to $+\infty$. Therefore, we shall assume the following.

ASSUMPTION (A3). For each $x, y \in R_{+}$, and $t \in[0, T]$ we have

$$
E\left[\sup _{t \in[0, T] \cap Q} \sup _{n} \bar{V}_{n}\left(x, y, \underline{s}_{t}, \bar{s}_{t}, t\right)\right]<\infty .
$$

We have

PROPOSITION 4.3. Under the assumption (A3) the processes $(V(x, y, t))$ and $\left(\bar{V}\left(x, y, \underline{s}_{t}, \bar{s}_{t}, t\right)\right)$ for $(x, y) \in R_{+}^{2}$ and $t \in[0, T]$ are finite and are solutions to the following system of equations:

$$
\begin{gathered}
\bar{V}\left(x, y, \underline{s}_{t}, \bar{s}_{t}, t\right)=\underset{(l, m) \in \mathcal{A}_{t}\left(x, y, \underline{s}_{t}, \bar{s}_{t}\right)}{\operatorname{ess} \sup _{t}} V\left(x+m \underline{s}_{t}-l \bar{s}_{t}, y-m+l, t\right), \\
V(x, y, t)=\underset{t \leqslant \tau \leqslant T}{\operatorname{ess} \sup } E\left[\bar{V}\left(x, y, \underline{s}_{\tau}, \bar{s}_{\tau}, \tau\right) \mid F_{t}\right] .
\end{gathered}
$$

Furthermore, for each $(x, y) \in Q_{+}^{2}$ the process $(V(x, y, t))$ is indistinguishable from a càdlàg process.

Proof. Notice that under (A3) we are allowed to enter ess sups with limit $r \rightarrow \infty$. For each $(x, y)$ the processes $\left(V_{r}(x, y, t)\right)$ are right continuous supermartingales so that, by Theorem VI.18 of [5], the process $(V(x, y, t))$ is indistinguishable from a càdlàg process. 
REMARK 4.1. In the case of continuous time we have to extend the class of controls to finite variation controls. The problem in the case of power or logarithmic utility leads to singular control problems as one can see for the infinite horizon case for lognormal prices in [U] and references therein. The control consisting of a finite number of transactions can be considered only as a potential approximation of the singular control problem. If we impose additional fixed costs for transactions, we can expect to find optimal control within the class of strategies consisting of a finite number of transactions, however the number of transactions will not be bounded deterministically from above. Under the assumption that we have a decision lag or execution delay, following each transaction decision (see [14]) we may expect to have optimal strategies consisting of a finite and bounded number of transactions. The last two approaches will be a subject of further work.

\section{APPENDIX}

We formulate here and prove some continuity results which are used in the paper. Denote by $h$ the Hausdorff metric defined on the space $H\left(R_{+}^{4}\right)$ of compact subsets of $R_{+}^{4}$ as follows:

$$
h(A, B):=\max \{d(A, B), d(B, A)\}
$$

with $d(A, B):=\sup \{\operatorname{dist}(a, B): a \in A\}$ and $\operatorname{dist}(x, A):=\inf \{\operatorname{dist}(x, a): a \in A\}$. Clearly, $\left(H\left(R_{+}^{4}\right), h\right)$ is a complete metric space (see, e.g., [3]). We have, by Theorem 2.1 of [1]3], the following

LEMMA 5.1. Let $\left(x_{n}, y_{n}, \underline{s}_{n}, \bar{s}_{n}\right)$ be a sequence with coordinates in $R_{+}^{4}$ such that $\underline{s}_{n} \leqslant \bar{s}_{n}$, which converges to $(x, y, \underline{s}, \bar{s})$, where $\underline{s}<\bar{s}$. Then

$$
h\left(A(x, y, \underline{s}, \bar{s}), A\left(x_{n}, y_{n}, \underline{s}_{n}, \bar{s}_{n}\right)\right) \stackrel{n \rightarrow \infty}{\longrightarrow} 0 .
$$

LeMma 5.2. Assume that $r_{t}^{m}(x, y)$ is a family of càdlàg trajectories depending on $(x, y) \in R_{+}^{2}$ and $m=1,2 \ldots$, i.e. for each $m$ the mapping $[0, T] \ni t \mapsto$ $r_{t}^{m}(x, y)$ is càdlàg, and furthermore, for each $m=1,2, \ldots$ the mapping $R_{+}^{2} \ni$ $(x, y) \mapsto r_{t}^{m}(x, y)$ is continuous uniformly in $t$ for $(x, y)$ from compact subsets in $R_{+}^{2}$. If for any compact set $K \subset R_{+}^{2}$ there is $r_{t}(x, y)$ for $(x, y) \in K \cap Q^{2}$ and $t \in[0, T] \cap Q$ such that

$$
\sup _{t \in[0, T] \cap Q} \sup _{(x, y) \in K \cap Q^{2}}\left|r_{t}^{m}(x, y)-r_{t}(x, y)\right| \rightarrow 0
$$

as $m \rightarrow \infty$, then $r_{t}(x, y):=\lim _{[0, T] \cap Q \ni t_{n} \downarrow t, Q^{2} \cap R_{+}^{2} \ni\left(x_{n}, y_{n}\right) \rightarrow(x, y)} r_{t_{n}}\left(x_{n}, y_{n}\right) d e$ fined for each $(x, y) \in R_{+}^{2}$ is such that the mapping $[0, T] \ni t \mapsto r_{t}(x, y)$ is càdlàg and the mapping $R_{+}^{2} \ni(x, y) \mapsto r_{t}(x, y)$ is continuous uniformly in $t$ for $(x, y)$ from compact subsets of $R_{+}^{2}$. 
Proof. For given $\varepsilon>0$ and a compact set $K \subset R_{+}^{2}$ there is $M(K, \varepsilon)$ such that for $m \geqslant M(K, \varepsilon)$ we have

$$
\sup _{t \in[0, T] \cap Q} \sup _{(x, y) \in K \cap Q^{2}}\left|r_{t}^{m}(x, y)-r_{t}(x, y)\right| \leqslant \varepsilon .
$$

Notice first that $r_{t}(x, y)$ is well defined for any $(x, y) \in R_{+}^{2}$ and $t \in[0, T]$. In fact, if $K \cap Q^{2} \ni\left(x_{n}, y_{n}\right),\left(x_{n}^{\prime}, y_{n}^{\prime}\right) \rightarrow(x, y),[0, T] \cap Q \ni t_{n} \downarrow t,[0, T] \cap Q \ni t_{n}^{\prime} \downarrow t$ and $\bar{r}_{t}(x, y)=\lim _{n \rightarrow \infty} r_{t_{n}}\left(x_{n}, y_{n}\right), \tilde{r}_{t}(x, y)=\lim _{n \rightarrow \infty} r_{t_{n}^{\prime}}\left(x_{n}^{\prime}, y_{n}^{\prime}\right)$, then by (5.3) and continuity of $R_{+}^{2} \ni(x, y) \mapsto r_{t}^{m}(x, y)$ for $m \geqslant M(K, \varepsilon)$ we have

$$
\begin{aligned}
\mid r_{t_{n}}\left(x_{n}, y_{n}\right) & -r_{t_{n}^{\prime}}\left(x_{n}^{\prime}, y_{n}^{\prime}\right) \mid \\
\leqslant & \left|r_{t_{n}}\left(x_{n}, y_{n}\right)-r_{t_{n}}^{m}\left(x_{n}, y_{n}\right)\right| \\
& +\left|r_{t_{n}}^{m}\left(x_{n}, y_{n}\right)-r_{t_{n}^{\prime}}^{m}\left(x_{n}^{\prime}, y_{n}^{\prime}\right)\right|+\left|r_{t_{n}^{\prime}}^{m}\left(x_{n}^{\prime}, y_{n}^{\prime}\right)-r_{t_{n}^{\prime}}\left(x_{n}^{\prime}, y_{n}^{\prime}\right)\right| \\
\leqslant & 2 \varepsilon+\left|r_{t_{n}}^{m}\left(x_{n}, y_{n}\right)-r_{t_{n}^{\prime}}^{m}\left(x_{n}^{\prime}, y_{n}^{\prime}\right)\right| \rightarrow 2 \varepsilon,
\end{aligned}
$$

letting $n \rightarrow \infty$, which means that $\bar{r}_{t}(x, y)=\tilde{r}_{t}(x, y)$. Furthermore, (5.3) and (5.2) hold for any $(x, y) \in K$ and $t \in[0, T]$. If $K \ni\left(x_{n}, y_{n}\right) \rightarrow(x, y)$, then

$$
\begin{aligned}
\sup _{t \in[0, T]} \mid r_{t}\left(x_{n}, y_{n}\right)- & r_{t}(x, y) \mid \\
\leqslant & \sup _{t \in[0, T]}\left\{\left|r_{t}\left(x_{n}, y_{n}\right)-r_{t}^{m}\left(x_{n}, y_{n}\right)\right|\right. \\
& \left.+\left|r_{t}^{m}\left(x_{n}, y_{n}\right)-r_{t}^{m}(x, y)\right|+\left|r_{t}^{m}(x, y)-r_{t}(x, y)\right|\right\} \\
\leqslant & 2 \varepsilon+\sup _{t \in[0, T]}\left\{\left|r_{t}^{m}\left(x_{n}, y_{n}\right)-r_{t}^{m}(x, y)\right|\right\},
\end{aligned}
$$

letting first $n \rightarrow \infty$, then $m \rightarrow \infty$, since $\varepsilon$ could be chosen arbitrarily small. Consequently, we infer that for each compact set $K$ we have uniform in $t$ continuity of $(x, y) \mapsto r_{t}(x, y)$. The càdlàg property of $t \mapsto r_{t}(x, y)$ follows now directly from convergence (5.2).

LEMMA 5.3. Assume that the mappings $[0, T] \ni t \mapsto \underline{s}_{t}$ and $[0, T] \ni t \mapsto \bar{s}_{t}$ are càdlàg and $\underline{s}_{t}<\bar{s}_{t}$ for $t \in[0, T]$. Assume furthermore that the mapping $R_{+}^{2} \ni$ $(x, y) \mapsto V(x, y, t)$ is continuous uniformly in $t$ for $(x, y)$ from compact sets, while the mapping $[0, T] \ni t \mapsto V(x, y, t)$ is càdlàg for each $(x, y) \in R_{+}^{2}$. Then

$$
\bar{V}\left(x, y, \underline{s}_{t}, \bar{s}_{t}, t\right):=\sup _{(l, m) \in A\left(x, y, \underline{s}_{t}, \bar{s}_{t}\right)} V\left(x+m \underline{s}_{t}-l \bar{s}_{t}, y-m+l, t\right)
$$

is continuous in $(x, y)$ from compact subsets in $R_{+}^{2}$ uniformly in $t$, and the mapping $[0, T] \ni t \mapsto \bar{V}\left(x, y, \underline{s}_{t}, \bar{s}_{t}, t\right)$ is càdlàg. 
Proof. Since $A(x, y, \underline{s}, \bar{s})$ is compact and $V(x, y-m+l, t)$ is continuous in $(x, y)$, there is $(l, m) \in A(x, y, \underline{s}, \bar{s})$ such that

$$
\bar{V}(x, y, \underline{s}, \bar{s}, t)=V(x+m \underline{s}-l \bar{s}, y-m+l, t) .
$$

Let now $R_{+}^{2} \ni\left(x_{n}, y_{n}\right) \rightarrow(x, y)$ and $\left(l_{n}, m_{n}\right) \in A\left(x_{n}, y_{n}, \underline{s}_{t}, \bar{s}_{t}\right)$ be such that

$$
\bar{V}\left(x_{n}, y_{n}, \underline{s}_{t}, \bar{s}_{t}, t\right)=V\left(x+m_{n} \underline{s}_{t}-l_{n} \bar{s}_{t}, y-m_{n}+l_{n}, t\right) .
$$

Without loss of generality, by Lemma 5.1 , choosing a suitable subsequence, we may assume that $\left(l_{n}, m_{n}\right) \rightarrow(l, m) \in A\left(x, y, \underline{s}_{t}, \bar{s}_{t}\right)$. Hence, by continuity,

$$
V\left(x_{n}+m_{n} \underline{s}_{t}-l_{n} \bar{s}_{t}, y_{n}-m_{n}+l_{n}, t\right) \rightarrow V\left(x+m \underline{s}_{t}-l \bar{s}_{t}, y-m+l, t\right)
$$

uniformly in $t \in[0, T]$, since trajectories of $\left(\underline{s}_{t}\right)$ and $\left(\bar{s}_{t}\right)$ take values in a compact set. Then

$$
\bar{V}\left(x, y, \underline{s}_{t}, \bar{s}_{t}, t\right) \geqslant V\left(x+m \underline{s}_{t}-l \bar{s}_{t}, y-m+l, t\right) .
$$

If there is $\left(l^{\prime}, m^{\prime}\right) \in A\left(x, y, \underline{s}_{t}, \bar{s}_{t}\right)$ such that

$$
V\left(x+m^{\prime} \underline{s}_{t}-l^{\prime} \bar{s}_{t}, y-m^{\prime}+l^{\prime}, t\right)>V\left(x+m \underline{s}_{t}-l \bar{s}_{t}, y-m+l, t\right),
$$

then by Lemma $[$.$] there is \left(l_{n}^{\prime}, m_{n}^{\prime}\right) \in A\left(x_{n}, y_{n}, \underline{s}_{t}, \bar{s}_{t}\right)$ such that $\left(l_{n}^{\prime}, m_{n}^{\prime}\right) \rightarrow$ $(l, m)$ and

$$
V\left(x_{n}+m_{n}^{\prime} \underline{s}_{t}-l_{n}^{\prime} \bar{s}_{t}, y_{n}-m_{n}^{\prime}+l_{n}^{\prime}, t\right) \rightarrow V\left(x+m^{\prime} \underline{s}_{t}-l^{\prime} \bar{s}_{t}, y-m^{\prime}+l^{\prime}, t\right) .
$$

Then

$$
V\left(x_{n}+m_{n}^{\prime} \underline{s}_{t}-l_{n}^{\prime} \bar{s}_{t}, y_{n}-m_{n}^{\prime}+l_{n}^{\prime}, t\right) \leqslant \bar{V}\left(x_{n}, y_{n}, \underline{s}_{t}, \bar{s}_{t}, t\right),
$$

and consequently

$$
V\left(x+m^{\prime} \underline{s}_{t}-l^{\prime} \bar{s}_{t}, y-m^{\prime}+l^{\prime}, t\right) \leqslant V\left(x+m \underline{s}_{t}-l \bar{s}_{t}, y-m+l, t\right),
$$

which is a contradiction to (5.6). Therefore, by (5.5) we have $\bar{V}\left(x_{n}, y_{n}, \underline{s}_{t}, \bar{s}_{t}, t\right) \rightarrow$ $\bar{V}\left(x, y, \underline{s}_{t}, \bar{s}_{t}, t\right)$ uniformly in $t \in[0, T]$.

Assume now that $t_{n} \downarrow t \in[0, T]$. Fix $(x, y) \in R_{+}^{2}$. There is $\left(l_{n}, m_{n}\right) \in A$ $\left(x, y, \underline{s}_{t_{n}}, \bar{s}_{t_{n}}\right)$ such that

$$
\bar{V}\left(x, y, \underline{s}_{t_{n}}, \bar{s}_{t_{n}}, t_{n}\right)=V\left(x+m_{n} \underline{s}_{t_{n}}-l_{n} \bar{s}_{t_{n}}, y-m_{n}+l_{n}, t_{n}\right) .
$$

Using Lemma [.] again, we may assume, taking into account right continuity of the trajectories of $\left(\underline{s}_{t}\right)$ and $\left(\bar{s}_{t}\right)$, that $\left(l_{n}, m_{n}\right) \rightarrow(l, m) \in A\left(x, y, \underline{s}_{t}, \bar{s}_{t}\right)$. We have

$$
\begin{aligned}
& \left|V\left(x+m_{n} \underline{s}_{t_{n}}-l_{n} \bar{s}_{t_{n}}, y-m_{n}+l_{n}, t_{n}\right)-V\left(x+m \underline{s}_{t}-l \bar{s}_{t}, y-m+l, t\right)\right| \\
\leqslant & \left|V\left(x+m_{n} \underline{s}_{t_{n}}-l_{n} \bar{s}_{t_{n}}, y-m_{n}+l_{n}, t_{n}\right)-V\left(x+m \underline{s}_{t}-l \bar{s}_{t}, y-m+l, t_{n}\right)\right| \\
& +\left|V\left(x+m_{s_{t}}-l \bar{s}_{t}, y-m+l, t_{n}\right)-V\left(x+m \underline{s}_{t}-l \bar{s}_{t}, y-m+l, t_{n}\right)\right| \\
= & a_{n}+b_{n}
\end{aligned}
$$


and $a_{n} \rightarrow 0$ as $n \rightarrow \infty$, by right continuity of the trajectories of $\left(\underline{s}_{t}\right)$ and $\left(\bar{s}_{t}\right)$ and uniform in $t$ continuity of $V$ with respect to the first two coordinates taking values in compact sets. Also, $b_{n} \rightarrow 0$ as $n \rightarrow \infty$, by right continuity of $[0, T] \ni$ $t \mapsto V(x, y, t)$ for any fixed $(x, y) \in R_{+}^{2}$. Consequently,

$$
\limsup _{n \rightarrow \infty} \bar{V}\left(x, y, \underline{s}_{t_{n}}, \bar{s}_{t_{n}}, t_{n}\right) \leqslant \bar{V}\left(x, y, \underline{s}_{t}, \bar{s}_{t}, t\right) .
$$

Suppose now we have in (5.7) a strict inequality. Let $(l, m) \in A\left(x, y, \underline{s}_{t}, \bar{s}_{t}\right)$ be such that

$$
\bar{V}\left(x, y, \underline{s}_{t}, \bar{s}_{t}, t\right)=V\left(x+m \underline{s}_{t}-l \bar{s}_{t}, y-m+l, t\right) .
$$

By Lemma [.], there is a sequence $\left(l_{n}, m_{n}\right) \in A\left(x, y, \underline{s}_{t_{n}}, \bar{s}_{t_{n}}\right)$ such that $\left(l_{n}, m_{n}\right)$ $\rightarrow(l, m)$, and therefore

$$
V\left(x+m_{n} \underline{s}_{t_{n}}-l_{n} \bar{s}_{t_{n}}, y-m_{n}+l_{n}, t_{n}\right) \rightarrow V\left(x+m \underline{s}_{t}-l \bar{s}_{t}, y-m+l, t\right) .
$$

Since for every $n$

$$
V\left(x+m_{n} \underline{s}_{t_{n}}-l_{n} \bar{s}_{t_{n}}, y-m_{n}+l_{n}, t_{n}\right) \leqslant \bar{V}\left(x, y, \underline{s}_{t_{n}}, \bar{s}_{t_{n}}, t_{n}\right),
$$

we have equality in (5.7) and instead of lim sup we have just lim there. This means that $\bar{V}$ is right continuous in $t$. To show that it has also the left-hand limit, it remains to show that for $t_{n} \uparrow t$ and $(x, y) \in R_{+}^{2}$

$$
\begin{aligned}
\lim _{n \rightarrow \infty} \bar{V}\left(x, y, \underline{s}_{t_{n}}, \bar{s}_{t_{n}}, t_{n}\right) & \\
& \rightarrow \sup _{(l, m) \in A\left(x, y, \underline{s}_{t}^{-}, \bar{s}_{t}^{-}\right)} V^{-}\left(x+m \underline{s}_{t}^{-}-l \bar{s}_{t}^{-}, y-m+l, t\right)
\end{aligned}
$$

as $n \rightarrow \infty$, where $\underline{s}_{t}^{-}, \bar{s}_{t}^{-}$are left limits of $\left(\underline{s}_{t}\right),\left(\bar{s}_{t}\right)$ at time $t$ and $V^{-}(x, y, t)$ denotes the left limit of $V(x, y, t)$ at time $t$. The proof is similar to that of right continuity and is left to the reader.

\section{REFERENCES}

[1] M. Akian, A. Sulem, and M. Taksar, Dynamic optimization of long-term growth rate for a proportional transaction costs and logarithmic utility, Math. Finance 11 (2) (2001), pp. $153-188$.

[2] J.-M. Bismut and B. Skalli, Temps d'arrêt optimal, théorie générale des processes et processus de Markov, Z. Wahrsch. Verw. Gebiete 39 (1977), pp. 301-313.

[3] C. Castaing and M. Valadier, Convex Analysis and Measurable Multifunctions, Lecture Notes in Math., Vol. 580, Springer, Berlin 1977.

[4] C. Dellacherie, Capacités et processus stochastiques, Springer, Berlin 1972.

[5] C. Dellacherie and P.-A. Meyer, Probabilities and Potential B: Theory of Martingales, North Holland, Amsterdam 1982. 
[6] C. Doléans-Dade, Intégrales stochastiques dépendant d'un paramètre, Publ. Inst. Statist. Univ. Paris 16 (1967), pp. 23-34.

[7] A. G. Fakeev, The optimal stopping of random processes with continuous time, Teor. Veroyatn. Primen. 15 (1970), pp. 336-344.

[8] S. He, J. Wang, and J. Yan, Semimartingale Theory and Stochastic Calculus, CRC Press, Boca Raton 1992.

[9] I. Karatzas and S. E. Shreve, Brownian Motion and Stochastic Calculus, second edition, Springer, New York 2000.

[10] P. D. Lax, Functional Analysis, Wiley, New York 2002.

[11] I. Norros, E. Valkeila, and J. Virtamo, An elementary approach to a Girsanov formula and other analytical results on fractional Brownian motions, Bernoulli 5 (4) (1999), pp. 571587.

[12] G. Peskir and A. N. Shiryaev, Optimal Stopping and Free-Boundary Problems, Birkhäuser, Basel 2006.

[13] T. Rogala and L. Stettner, On construction of discrete time shadow price, AMO 72 (3) (2015), pp. 391-433.

[14] L. Stettner. Asymptotics of HARA utility from terminal wealth under proportional transaction costs with decision lag or execution delay and obligatory diversification, in: Advanced Mathematical Methods for Finance, G. Di Nunno and B. Øksendal (Eds.), Springer, BerlinHeidelberg 2011, pp. 509-536.

[15] C. Stricker and M. Yor, Calcul stochastique dépendant d'un paramètre, Z. Wahrsch. Verw. Gebiete 45 (1978), pp. 109-133.

Tomasz Rogala

Institute of Mathematics

Polish Academy of Sciences

ul. Śniadeckich 8

00-656 Warsaw, Poland

E-mail:trogala@op.pl
Łukasz Stettner Institute of Mathematics Polish Academy of Sciences (also Vistula University) ul. Śniadeckich 8 00-656 Warsaw, Poland E-mail: stettner@impan.pl

Received on 29.6.2016;

revised version on 6.12 .2016 